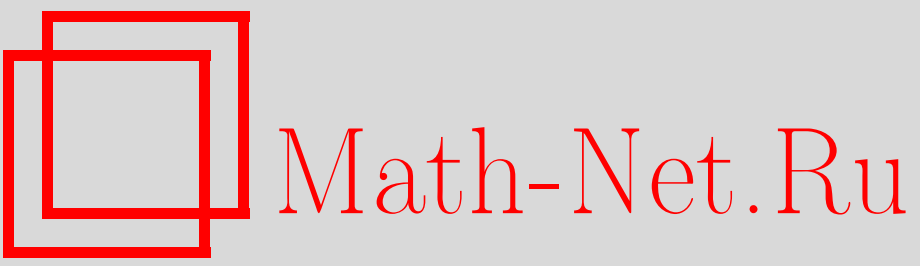

Н. В. Бейлина, О разрешимости обратной задачи для гиперболического уравнения с интегральным условием переопределения, Вестн. Сам. гос. техн. ун-та. Сер. Физ.-мат. науки, 2011, выпуск 2(), 34-39

DOI: https://doi.org/10.14498/vsgtu957

Использование Общероссийского математического портала Math-Net.Ru подразумевает, что вы прочитали и согласны с пользовательским соглашением

http://www. mathnet.ru/rus/agreement

Параметры загрузки:

IP: 54.80 .97 .219

26 апреля 2023 г., 18:37:20 
УДК 517.956.37

\section{О РАЗРЕШИМОСТИ ОБРАТНОЙ ЗАДАЧИ ДЛЯ ГИПЕРБОЛИЧЕСКОГО УРАВНЕНИЯ С ИНТЕГРАЛЬНЫМ УСЛОВИЕМ ПЕРЕОПРЕДЕЛЕНИЯ}

\section{Н. В. Бейлина}

Самарский государственный технический университет, 443100, Самара, ул. Молодогвардейская, 244.

E-mails: natalie@samdiff.ru

Изучается разрешимость обратной задачи для гиперболического уравнения на плоскости с неизвестной правой частъю. Доказывается однозначная разрешимость поставленной задачи. Доказательство существования обобщённого решения базируется на методе Галёркина, единственности - на полученной априорной оченке.

Ключевые слова: обратная задача, интегральное условие, разрешимостъ.

Введение. K настоящему времени появилось значительное количество работ, посвященных исследованию обратных задач с интегральным условием переопределения. Однако в подавляющем большинстве изучены задачи для параболических уравнений [1-7]. Более подробная библиография и классификация задач приведена в работах $[5,7]$.

В предлагаемой работе рассмотрена обратная задача нахождения неизвестной функции, входящей в правую часть гиперболического уравнения, с интегральным условием переопределения.

1. Постановка задачи. В прямоугольнике $Q_{T}=\{(x, t): 0<x<l, 0<t<T\}$ рассмотрим следующую обратную задачу: найти пару функций $(u(x, t), p(t))$, удовлетворяющих уравнению

$$
u_{t t}(x, t)-u_{x x}(x, t)+c(x, t) u(x, t)=p(t) f(x, t)+G(x, t, u(x, t)),
$$

начальным условиям

$$
u(x, 0)=\varphi(x), \quad u_{t}(x, 0)=\psi(x),
$$

граничным условиям

$$
u_{x}(0, t)=u_{x}(l, t)=0
$$

и интегральному условию переопределения

$$
\int_{0}^{l} K(x, t) u(x, t) d x=0 .
$$

Функции $\varphi(x), \psi(x)$ заданы на отрезке $[0 ; l]$, a $f(x, t), K(x, t), G(x, t, u)$, $c(x, t)$ - в области $\bar{Q}_{T}$.

Введём понятие обобщённого решения задачи (1)-(4). Заметим, что условие (4) эквивалентно следующему условию:

Бейлина Наталъя Викторовна (к.ф.-м.н.), старший преподаватель, каф. высшей математики и прикладной информатики. 


$$
\begin{aligned}
p(t)=\left(\int_{0}^{l} K(x, t) f(x, t) d x\right)^{-1}\left[\int_{0}^{l}\left[K(x, t) c(x, t)-K_{t t}(x, t)\right] u(x, t) d x+\right. \\
+\int_{0}^{l} K_{x}(x, t) u_{x}(x, t) d x-2 \int_{0}^{l} K_{t}(x, t) u_{t}(x, t) d x- \\
\left.\quad-\int_{0}^{l} K(x, t) G(x, t, u(x, t)) d x\right] .
\end{aligned}
$$

Действительно, дифференцируя (4) дважды по $t$ и учитывая, что $u(x, t)$ удовлетворяет уравнению (1) и условию (3), получим (5). Обратное показывается прямым вычислением.

Обозначим

$$
\hat{W}_{2}^{1}\left(Q_{T}\right)=\left\{v(x, t): v(x, t) \in W_{2}^{1}\left(Q_{T}\right), v(x, T)=0\right\} .
$$

Умножим уравнение $(1)$ на функцию $v(x, t) \in \hat{W}_{2}^{1}\left(Q_{T}\right)$ и проинтегрируем по прямоугольнику $Q_{T}$. После интегрирования по частям получим

$$
\begin{array}{r}
\int_{0}^{T} \int_{0}^{l}\left[-u_{t}(x, t) v_{t}(x, t)+u_{x}(x, t) v_{x}(x, t)+c(x, t) u(x, t) v(x, t)\right] d x d t= \\
=\int_{0}^{T} \int_{0}^{l} p(t) f(x, t) v(x, t) d x d t+\int_{0}^{T} \int_{0}^{l} G(x, t, u(x, t)) v(x, t) d x d t+ \\
+\int_{0}^{l} \psi(x) v(x, 0) d x
\end{array}
$$

ОПредЕЛЕниЕ. Пару функций $(u(x, t), p(t))$ будем называть обобщённым решением задачи (1)-(4), если $u(x, t) \in W_{2}^{1}\left(Q_{T}\right), u(x, 0)=\varphi(x), p(t) \in L_{2}(0, T)$, и $(u(x, t), p(t))$ удовлетворяет (5) (в смысле равенства функций в $\left.L_{2}\right)$ и тождеству (6) для любой функции $v(x, t) \in \hat{W}_{2}^{1}\left(Q_{T}\right)$.

\section{2. Разрешимость поставленной задачи.}

Teоpema. Ecлu $K(x, t) \in C^{1}\left(\bar{Q}_{T}\right), K_{t t}(x, t) \in C^{1}\left(\bar{Q}_{T}\right), c(x, t) \in C^{1}\left(\bar{Q}_{T}\right)$, $f(x, t) \in C\left(\bar{Q}_{T}\right), \varphi(x) \in W_{2}^{1}(0, l), \psi(x) \in L_{2}(0, l), G(x, t, u) \in C\left(Q_{T} \times \mathbb{R}^{1}\right)$, $\int_{0}^{l} K(x, t) f(x, t) d x \neq 0$ и для любых $(x, t)$ выполняется условие Липшица $\left|G\left(x, t, u_{1}\right)-G\left(x, t, u_{2}\right)\right| \leqslant L\left|u_{1}-u_{2}\right|$, то существует единственное обобщённое решение (1)-(4).

Доказательство. Из условий теоремы следует, что найдутся такие положительные константы $c_{0}, c_{1}, c_{2}, f_{1}, g_{1}, h_{0}, a_{1}, a_{2}$, что выполняются следующие неравенства:

$$
\begin{gathered}
\max |G(x, t, 0)| \leqslant g_{1}, \quad|f(x, t)| \leqslant f_{1}, \quad \max \left|K(x, t) c(x, t)-K_{t t}(x, t)\right| \leqslant a_{1}, \\
\max \left|K(x, t) ; K_{t}(x, t) ; K_{x}(x, t)\right| \leqslant a_{2}, \quad c_{0} \leqslant|c(x, t)| \leqslant c_{1}, \quad\left|c_{t}(x, t)\right| \leqslant c_{2}, \\
\int_{0}^{l} K(x, t) f(x, t) d x=h_{0}>0 .
\end{gathered}
$$


Для доказательства теоремы построим последовательность приближённых решений, а затем покажем, что эта последовательность сходится к обобщённому решению поставленной задачи.

Приближённые решения $\left(u^{m}(x, t), p^{m}(t)\right)$ будем искать из следующих соотношений:

$$
\begin{gathered}
\int_{0}^{T} \int_{0}^{l}\left[-u_{t}^{m}(x, t) v_{t}(x, t)+u_{x}^{m}(x, t) v_{x}(x, t)+c(x, t) u^{m}(x, t) v(x, t)\right] d x d t= \\
=\int_{0}^{T} \int_{0}^{l} p^{m}(t) f(x, t) v(x, t) d x d t+\int_{0}^{T} \int_{0}^{l} G\left(x, t, u^{m}(x, t)\right) v(x, t) d x d t+ \\
+\int_{0}^{l} \psi(x) v(x, 0) d x \\
u^{0}(x, t)=0, \quad u^{m}(x, 0)=\varphi_{m}, \quad m=1,2, \ldots
\end{gathered}
$$

где $\varphi_{m} \stackrel{L_{2}}{\longrightarrow} \varphi(x)$

$$
\begin{gathered}
p^{m}(t)=\left(\int_{0}^{l} K(x, t) f(x, t) d x\right)^{-1}\left[\int_{0}^{l}\left[K(x, t) c(x, t)-K_{t t}(x, t)\right] \times\right. \\
\times u^{m-1}(x, t) d x+\int_{0}^{l} K_{x}(x, t) u_{x}^{m-1}(x, t) d x-2 \int_{0}^{l} K_{t}(x, t) u_{t}^{m-1}(x, t) d x- \\
\left.\quad-\int_{0}^{l} K(x, t) G\left(x, t, u^{m-1}\right) d x\right] .
\end{gathered}
$$

Покажем, что для каждого $m$ существует единственная функция $u^{m}(x, t)$, удовлетворяющая тождеству (7) и условию $(8)$, если $p^{m}(t)$ известно. Для этого заметим, что тождество (7) и равенство (8) определяют обобщённое решение из $W_{2}^{1}\left(Q_{T}\right)$ второй начально-краевой задачи с однородными граничными условиями

$$
\begin{gathered}
u_{t t}^{m}(x, t)-u_{x x}^{m}(x, t)+c(x, t) u^{m}(x, t)=H(x, t)+G\left(x, t, u^{m}(x, t)\right), \\
H(x, t)=p^{m}(t) f(x, t), \\
u^{m}(x, 0)=\varphi^{m}(x), \quad u_{t}^{m}(x, 0)=\psi(x), \\
u_{x}^{m}(0, t)=u_{x}^{m}(l, t)=0 .
\end{gathered}
$$

Применяя стандартные методы [8] и условие Липшица, которому удовлетворяет функция $G(x, t, u(x, t))$, нетрудно доказать однозначную разрешимость задачи (10)-(12). Следовательно, можно утверждать, что для любого $p^{m}(t)$ существует единственная функция $u^{m}(x, t)$, удовлетворяющая тождеству $(7)$. Так как $p^{m}(t)$ находятся явным образом из (9), то можно считать, что последовательность $\left\{u^{m}(x, t), p^{m}(t)\right\}$ построена.

Для обоснования сходимости этой последовательности рассмотрим разности

$$
z^{m}(x, t)=u^{m}(x, t)-u^{m-1}(x, t), r^{m}(t)=p^{m}(t)-p^{m-1}(t) .
$$

Заметим, что для $z^{m}(x, t)$ справедливо тождество 


$$
\begin{aligned}
& \int_{0}^{T} \int_{0}^{l}\left[z_{t t}^{m}(x, t) v(x, t)\right.+z_{x}^{m}(x, t) v_{x}(x, t)+ \\
&\left.+c(x, t) z^{m}(x, t) v(x, t)\right] d x d t=\int_{0}^{T} \int_{0}^{l} r^{m}(t) f(x, t) v(x, t) d x d t+ \\
& \quad+\int_{0}^{T} \int_{0}^{l}\left[G\left(x, t, u^{m}\right)-G\left(x, t, u^{m-1}\right)\right] v(x, t) d x d t
\end{aligned}
$$

Полагая $v(x, t)=z_{t}^{m}(x, t)$ и интегрируя по частям интегралы в левой части, получим

$$
\begin{gathered}
\int_{0}^{l}\left[\left(z_{t}^{m}(x, \tau)\right)^{2}+\left(z_{x}^{m}(x, \tau)\right)^{2}+c(x, \tau)\left(z^{m}(x, \tau)\right)^{2}\right] d x= \\
=2 \int_{0}^{\tau} \int_{0}^{l} r^{m}(t) f(x, t) z_{t}^{m}(x, t) d x d t+2 \int_{0}^{\tau} \int_{0}^{l} c_{t}(x, t)\left(z_{t}^{m}(x, t)\right)^{2} d x d t+ \\
\quad+2 \int_{0}^{\tau} \int_{0}^{l}\left[G\left(x, t, u^{m}\right)-G\left(x, t, u^{m-1}\right)\right] z_{t}^{m}(x, t) d x d t .
\end{gathered}
$$

Применяя к правой части неравенство Юнга, элементарные неравенства, а также условия теоремы, нетрудно получить оценку

$$
\begin{gathered}
m \int_{0}^{l}\left[\left(z_{t}^{m}(x, \tau)\right)^{2}+\left(z_{x}^{m}(x, \tau)\right)^{2}+\left(z^{m}(x, \tau)\right)^{2}\right] d x \leqslant \\
\leqslant \delta\left(1+L^{2}\right) \int_{0}^{\tau} \int_{0}^{l}\left(r^{m}(t)\right)^{2} d x d t+ \\
+M_{3} \int_{0}^{\tau} \int_{0}^{l}\left(\left(z_{t}^{m}(x, t)\right)^{2}+\left(z^{m}(x, t)\right)^{2}+\left(z_{x}^{m}(x, t)\right)^{2}\right] d x d t
\end{gathered}
$$

где $M_{3}=\max \left\{\left(f_{1}^{2}+1\right) / \delta ; 2 c_{2}\right\}$. Применим к (13) неравенство Гронуолла [9], а затем интегрируя по $\tau$ от 0 до $T$, приходим к оценке

$$
\left\|z^{m}\right\|_{W_{2}^{1}(Q)} \leqslant \sqrt{N_{1} \delta}\left\|r^{m}(t)\right\|_{L_{2}(0, T)},
$$

где $N_{1}=\exp \left(\frac{M_{3} T}{m}\right)\left(1+L^{2}\right), \delta>0$ произвольно.

Оценим теперь $r^{m}(t)$, для которого справедливо равенство

$$
\begin{aligned}
r^{m}(t)= & \left(\int_{0}^{l} K(x, t) f(x, t) d x\right)^{-1}\left[\int_{0}^{l}\left[K(x, t) c(x, t)-K_{t t}(x, t)\right] z^{m-1}(x, t) d x+\right. \\
& +\int_{0}^{l} K_{x}(x, t) z_{x}^{m-1}(x, t) d x-2 \int_{0}^{l} K_{t}(x, t) z_{t}^{m-1}(x, t) d x- \\
& \left.-\int_{0}^{l} K(x, t)\left[G\left(x, t, u^{m-1}\right)-G\left(x, t, u^{m-2}\right)\right] d x\right]
\end{aligned}
$$

Возводя равенство (15) в квадрат, учитывая условия теоремы, а также то, что $z^{m-1}(x, t) \in W_{2}^{1}\left(Q_{T}\right)$, нетрудно получить неравенство

$$
\left.\| r^{m}(t)\right)\left\|_{L_{2}(0, T)} \leqslant \sqrt{N_{2}}\right\| z^{m-1}(x, t) \|_{W_{2}^{1}\left(Q_{T}\right)},
$$


где $N_{2}=\max \left\{4 a_{2}^{2} l ; 4\left(a_{1}^{2} l+a_{2}^{2} l L^{2}\right)\right\}$.

Из (14) и (16) вытекает, что

$$
\begin{gathered}
\left.\| z^{m}(x, t)\right)\left\|_{W_{2}^{1}(0, T)} \leqslant \sqrt{N_{3}}\right\| z^{m-1}(x, t) \|_{W_{2}^{1}\left(Q_{T}\right)}, \\
\left.\left.\| r^{m}(t)\right)\left\|_{L_{2}(0, T)} \leqslant \sqrt{N_{3}}\right\| r^{m-1}(t)\right) \|_{L_{2}(0, T)}
\end{gathered}
$$

где $N_{3}=N_{1} N_{2} \delta$.

Пользуясь произволом $\delta$, выберем его так, чтобы $\sqrt{N_{3}}=q<1$. Тогда неравенства (17) и (18) означают, что последовательность $\left(u^{m}(x, t), p^{m}(t)\right)$ фундаментальна.

Так как $W_{2}^{1}\left(Q_{T}\right)$ и $L_{2}(0, T)$ - полные пространства, то фундаментальная последовательность $\left(u^{m}(x, t), p^{m}(t)\right)$ сходится к элементу $(u(x, t), p(t))$, где $u(x, t) \in W_{2}^{1}\left(Q_{T}\right), p(t) \in L_{2}(0, T)$. Но тогда, переходя к пределу в $(7)$ и $(9)$, получим соответственно тождества (6) и (5), так как из сильной сходимости следует слабая.

Таким образом, пара функций $(u(x, t), p(t))$, полученная в результате предельного перехода в $\left(u^{m}(x, t), p^{m}(t)\right)$ и эквивалентных преобразований, является обобщённым решением задачи (1)-(4).

Единственность задачи (1)-(4) непосредственным образом следует из оценок (17) и (18). Действительно, полагая, что существуют два различных решения $\left(u_{1}, p_{1}\right)$ и $\left(u_{2}, p_{2}\right)$, приходим к оценкам

$$
(1-q)\|u(x, t)\|_{W_{2}^{1}\left(Q_{T}\right)} \leqslant 0, \quad(1-q)\|p(t)\|_{L_{2}(0, T)} \leqslant 0,
$$

где $(u, p)=\left(u_{1}-u_{2}, p_{1}-p_{2}\right)$. Но так как $q<1$, то $\|u(x, t)\|_{W_{2}^{1}\left(Q_{T}\right)}=0$ и $\|p(t)\|_{L_{2}(0, T)}=0$.

Работа выполнена в рамках ФЦП «Научные и научно-педагогические кадры инновационной России» на 2009-2013 годы, в рамках мероприятия 1.3.1 (госконтракт № П2589 от 26.11.2009)

\section{БИБЛИОГРАФИЧЕСКИЙ СПИСОК}

1. Cannon J. R., Lin $Y$. Determination of a control parameter in a parabolic partial differential equation // J. Austral. Math. Soc. Ser. B, 1991. Vol.33, no. 2. Pp. 149-163.

2. Cannon J.R., Lin Y. Determination of a parameter $p(t)$ in some quasi-linear parabolic differential equations // Inverse Problems, 1998. Vol. 4, no. 1. Pp. 35-45.

3. Иванчов Н.И. Об определении зависящего от времени старшего коэффициента в параболическом уравнении // Сиб. матем. журн., 1998. Т. 39, №3. С. 539-550; англ. пер.: Ivanchov N.I. On the determination of the time-dependent leading coefficient in a parabolic equation // Siberian Math. J., 1998. Vol. 39, no. 3. Pp. 465-475.

4. Камынин В. Л. Об обратной задаче определения правой части в параболическом уравнении с условием интегрального переопределения // Матем. заметки, 2005. Т. 77, № 4. C. 522-534; англ. пер.: Kamynin V. L. On the inverse problem of determining the right-hand side of a parabolic equation under an integral overdetermination condition// Math. Notes, 2005. Vol. 77, no. 4. Pp. 482-493.

5. Кожанов А. И. О разрешимости обратной задачи нахождения коэффициента теплопроводности // Сиб. матем. журн., 2005. Т. 46, № 5. С. 1053-1071; англ. пер.: Kozhanov A. I. Solvability of the inverse problem of finding thermal conductivity // Siberian Math. J., 2005. Vol. 46, no. 5. Pp. 841-856. 
6. Прилепко А.И., Костин А.Б. О некоторых обратных задачах для параболических уравнений с финальным и интегральным наблюдением // Матем. сб., 1992. Т. 183, № 4. C. 49-68; англ. пер.: Prilepko A.I., Kostin A. B. On certain inverse problems for parabolic equations with final and integral observation // Russian Acad. Sci. Sb. Math., 1993. Vol.75, no. 2. Pp. 473-490.

7. Прилепко А.И., Ткаченко Д. С. Свойства решений параболического уравнения и единственность решения обратной задачи об источнике с интегральным переопределением // Ж. вычисл. матем. и матем. физ., 2003. Т. 43, № 4. С. 562-570; англ. пер.: Prilepko A. I., Tkachenko D.S. Properties of solutions of a parabolic equation and the uniqueness of the solution of the inverse source problem with integral overdetermination // Comput. Math. Math. Phys., 2003. Vol. 43, no. 4. Pp. 537-546.

8. Ладыженская О. А., Солонников В. А., Уральщева Н. Н. Линейные и квазилинейные уравнения параболического типа. М.: Наука, 1967. 736 с.; англ. пер.: Ladyzenskaja O. A., Solonnikov V.A., Ural'ceva N.N. Linear and quasi-linear equations of parabolic type/ Translations of Mathematical Monographs. Vol. 23. Providence, R.I.: American Mathematical Society, 1968. 648 pp.

9. Gârding L. Cauchy's problem for hyperbolic equations / Lecture notes. Chicago, Ill, USA: University of Chicago, 1957; русск. пер.: Гординг Л. Задача Коши для гиперболических уравнений. М.: Иностр. лит., 1961. 120 с.

Поступила в редакцию 25/IV/2011;

в окончательном варианте - 05/V/2011.

MSC: 35R30; 35L10

\section{ON SOLVABILITY OF A INVERSE PROBLEM FOR HYPERBOLIC EQUATION WITH AN INTEGRAL OVERDETERMINATION CONDITION}

\section{N. V. Beilina}

Samara State Technical University,

244, Molodogvardeyskaya st., Samara, 443100, Russia.

E-mails: natalie@samdiff.ru

In this paper we study an inverse problem with an integral overdetermination condition for a hyperbolic equation with an unknown coefficient in equation. The existence and uniqueness of a solution is proved with the help of an a-priory estimate and Galyorkin procedure.

Key words: inverse problem, integral condition, solvability.

Original article submitted $25 / \mathrm{IV} / 2011$; revision submitted $05 / \mathrm{V} / 2011$.

Natalya V. Beilina (Ph. D. (Phys. \& Math.)), Senior Teacher, Dept. of Higher Mathematics \& Applied Informatics. 